\title{
BMJ Open A qualitative investigation into patients' views on visual field testing for glaucoma monitoring
}

\author{
Fiona C Glen, Helen Baker, David P Crabb
}

To cite: Glen FC, Baker H, Crabb DP. A qualitative investigation into patients' views on visual field testing for glaucoma monitoring. BMJ Open 2014;4:e003996. doi:10.1136/bmjopen-2013003996

- Prepublication history for this paper is available online. To view these files please visit the journal online (http://dx.doi.org/10.1136/ bmjopen-2013-003996).

FCG and $\mathrm{HB}$ are joint lead author.

Received 10 September 2013 Revised 20 November 2013 Accepted 10 December 2013

CrossMark

Division of Optometry and Visual Science, School of Health Sciences, City University London, London, UK

Correspondence to Professor David Crabb; david.crabb.1@city.ac.uk

\section{ABSTRACT \\ Objectives: To investigate the views and experiences of patients regarding their glaucoma follow-up, particularly towards the type and frequency of visual field (VF) testing.}

Design: A qualitative investigation using focus groups. The group discussion used broad open questions around the topics in a prompt guide relating to experiences of glaucoma follow-up, and in particular, VF monitoring. All the groups were taped, transcribed and coded using manual and computer-aided methods. Setting: Three National Health Service (NHS) hospitals in England; two focus groups took place at each hospital.

Participants: 28 patients (mean (SD) age: 74 (9) years; $54 \%$ women) diagnosed with glaucoma for at least 2 years. Each focus group consisted of 3-6 patients.

Primary and secondary outcomes: (1) Attitudes and experiences of patients with glaucoma regarding VF testing. (2) Patients' opinions about successful follow-up in glaucoma.

Results: These patients did not enjoy the VF test but they recognised the importance of regular monitoring for preserving their vision. These patients would agree to more frequent VF testing on their clinician's recommendation. A number of themes recurred throughout the focus groups representing perceived barriers to follow-up care. The testing environment, waiting times, efficiency of appointment booking and travel to the clinic were all perceived to influence the general clinical experience and the quality of assessment data. Patients were also concerned about aspects of patient-doctor communication, and often received little to no feedback about their results.

Conclusions: Patients trust the clinician to make the best decisions for their glaucoma follow-up. However, patients highlighted a number of issues that could compromise the effectiveness of VF testing. Addressing patient-perceived barriers could be an important step for devising optimal strategies for follow-up care.

\section{INTRODUCTION}

Glaucoma is a group of chronic diseases of the optic nerve that, if not managed effectively, could lead to visual impairment or

\section{Strengths and limitations of this study}

- This is the first qualitative study to examine patients' views of visual field monitoring using focus groups.

- Focus groups took place at three selected hospitals in the South of England; it is assumed that the views expressed represent the experiences of patients in a wider population.

- Not all patients approached by their ophthalmologist took part, but reasons for nonparticipation were not monitored. Patients who chose to volunteer may be more articulate, motivated and opinionated than the general patient population.

blindness. Currently, the only modifiable risk factor for disease worsening (progression) in glaucoma is reduction of intraocular pressure (IOP). A variety of different approaches to IOP lowering are available, meaning surveillance of the patient is important in selecting the correct intensity of treatment. Over half a million people in the UK are thought to have the condition, with clinics receiving over a million outpatient visits annually. ${ }^{12}$ Since the prevalence of glaucoma increases exponentially with age, these figures can be expected to increase dramatically with an ageing population. Glaucoma monitoring, therefore, represents a major workload for eye services in the National Health Service (NHS).

Assessment of non-seeing or 'blind' areas of the visual field (VF) is central to the monitoring of visual function in glaucoma. The $\mathrm{VF}$ is assessed by standard automated perimetry (SAP), a sophisticated automated instrument. The test is carried out in a darkened room and takes about $10 \mathrm{~min}$ per eye. In short, a patient looks into the part of the instrument that consists of a large semicircular bowl covering their entire field of view. The instrument presents a series of stimuli (spots of light), one at a time, at a range of contrast levels at varying locations in the $\mathrm{VF}$ 
while the patient fixates on a central point. The patient responds by clicking a button when a stimulus is detected. This process yields a map of the seeing parts of the patient's field of view; this map is subjected to statistical analysis comparing a patient's results to normative values for people with healthy vision. These measurements can be highly variable, and speed (rate) of VF loss, determined from a series of measurements over a period of time, varies considerably between treated individuals. The VF should, therefore, be monitored at appropriate intervals in order to identify timely intervention of more intensified treatment to preserve visual function. ${ }^{3}$ Yet, evidence regarding how frequently $\mathrm{VF}$ tests should be carried out to optimally detect disease progression is limited. The National Institute of Care Excellence (NICE) called for more research into examining the effectiveness of using different monitoring intervals to detect disease progression in people with glaucoma in 2009. ${ }^{1}$ Guidelines proposed by the European Glaucoma Society (EGS) recommend that the frequency of VF tests should be increased for newly diagnosed patients in order to better determine the speed of $\mathrm{VF}$ progression. This notion is supported by research evidence based on statistical analyses of retrospective data which has indicated that three VF tests per year in the first 2 years of follow-up would be clinically useful for identifying patients who are deteriorating at faster rates. ${ }^{4-6}$ However, a recent multicentre audit of glaucoma clinics in England indicated that most patients have only about one VF test a year. ${ }^{7}$ In another recent study, VF monitoring intervals assigned by clinicians (for hypothetical patient scenarios) were shown to be highly variable. $^{8}$

Organisational and resource constraints in the current NHS setting will impact on the feasibility of translating research-supported guidelines for $\mathrm{VF}$ monitoring to practice. Furthermore, the clinician ultimately drives decision-making based on their own estimates of the likelihood and speed of disease progression, and therefore their opinions towards the appropriateness of monitoring intervals will be important. At the same time, establishing effective monitoring strategies for this chronic condition likely also requires the input of the patients themselves, especially if it equates to more clinic visits. Care plans that place burdens on patients may result in a reduced willingness to return for follow-up and compromise the quality of the data obtained that is subsequently relied on during management. ${ }^{9}{ }^{10}$ Studies have shown that the views of the clinician and the patient regarding aspects of their condition are not always aligned, ${ }^{11} 12$ implying the patient's perspective must also be considered. Nevertheless, there has been limited use of patient-based research for improving glaucoma care. A review of the literature found that most of the studies use questionnaires to quickly gather information about the perceptions of patients, usually with regard to their perceived outcomes. ${ }^{13}$ However, questionnaires can be impersonal and subject to bias. ${ }^{14} 15$
Qualitative techniques, such as focus groups, offer an alternative method of gathering information about what a patient thinks and how they think or why they may hold a particular view. Group interaction encourages participants to explore and clarify individual and shared perspectives and supports the participation of people who may be reluctant to contribute their views in a more formal one-to-one scenario. ${ }^{16}$ Focus groups have been used in a small number of studies to examine the general experiences of glaucoma patients at diagnosis, their expectations and to identify potential barriers to treatment adherence. ${ }^{10}$ 17-19 However, there is limited evidence regarding the opinions of patients about the manner in which their vision loss is monitored. Anecdotal evidence suggests that patients dislike performing the $\mathrm{VF}$ test, and one quantitative study showed that patients rate the VF test least favourably of all the vision assessments. ${ }^{20}$ However, no study has interviewed patients with glaucoma in detail about their perceptions of the VF test and their follow-up care. The current study, therefore, aims to shed light on the effectiveness of glaucoma monitoring from the perspective of the patient by exploring patient's views and experiences via focus groups. In particular, the study aims to establish patients' views about VF testing in glaucoma monitoring.

\section{METHODS}

\section{Participants and methods}

Focus groups took place between May 2012 and January 2013 in the following locations: The Queen Alexandra Hospital NHS Trust in Portsmouth; Norfolk and Norwich University Hospital NHS Foundation Trust in Norwich and Moorfields Eye Hospital NHS Foundation Trust in London. The study was multicentred to reduce the bias that might come from one geographical area and to encompass healthcare trusts in urban and rural locations. The sites were chosen because they were involved in a wider programme work, of which the current study was a component. There were two focus groups at each site, with participants allocated to one of the two groups at the corresponding hospital.

The study used purposeful sampling whereby a consultant ophthalmologist at each participating eye hospital selected suitable participants during their routine eye check-up. Specifically, the participant was required to be aged 60 years and over and to be an established glaucoma patient who had been under review for at least 2 years. These criteria were chosen to reflect the age-related nature of disease and to ensure that participants had had sufficient experience of VFs as part of their glaucoma follow-up. The ophthalmologist gave potential participants an information sheet, and interested people were asked to sign a form indicating they were happy to be contacted by a researcher (it was stressed that they were not obliged to participate). Each consultant ophthalmologist approached 20 patients in this way. One of the study investigators (HB) then 
contacted the patients with further information and invited them to take part on one of the two specific dates at the corresponding hospital. Those who declined did so because they were not available on the specific dates (no other reason was cited). Initially, five to six patients were signed up to participate on each of the six study dates. However, a small number $(n=4)$ of patients did not attend. A total of 28 participants (mean age (SD) 74 (9) years; $54 \%$ women) eventually took part across the six focus groups. Each group consisted of three to six patients and included participants of both genders.

\section{Procedure}

A topic guide was devised prior to beginning the study, outlining broad question areas regarding general glaucoma care, experiences of the $\mathrm{VF}$ test and opinions about the VF test frequency. Study topics were informed by an initial pilot exercise involving a discussion with two patients with glaucoma, who also provided additional verbal and written information about their experiences. The topics included in the guide acted only as suggestions; the wording of questions was not predetermined and the order of the topics was not fixed. Prompts were used to introduce topic areas and encourage respondents to elaborate but the onus was on participants to supply the overall content of the discussion. Care was taken to ensure that the questions were open and 'nonleading', although more specific questioning was sometimes used to clarify a point made by a participant. If discussion went substantially off-topic, or one participant was dominating the conversation, the interviewer would reflect back to a previous topic and encourage other participants to contribute their views.

Prior to the study, participants were informed that they would be involved in "an open discussion about (their) experiences in the glaucoma clinic, with special attention to the visual tests (they) undertake". Participants were not explicitly aware of the emphasis on VF testing, so as to avoid bias linked to the self-selection of participants with strong views on this one topic. All focus groups were conducted by one of the authors (HB), a postdoctoral researcher who had a prior experience of qualitative research involving patients with glaucoma. ${ }^{18} 21$ The interviewer and participants had no prior knowledge of each other in a clinical or personal context, so each focus group began with general introductions. Field notes were taken during the sessions to aid later interpretation of the data, although note-taking was purposely minimal so that the interviewer could be fully attentive to the discussion. The focus groups lasted between 60 and $75 \mathrm{~min}$.

The study conformed to the Declaration of Helsinki and written consent from all participants was obtained prior to each focus group.

The study was designed and reported in accordance with the Consolidated Criteria for Reporting Qualitative Research (COREQ) for interviews and focus groups. ${ }^{22}$

\section{Analysis}

All focus groups were audiorecorded (with permission from the participants). The dialogue from the recordings was later transcribed and reviewed by the investigators. In a small number of instances, certain words were inaudible on the recordings due to excessive background noise, so field notes were used to account for any unclear information.

Data were analysed by two of the authors (HB and FCG) independently using framework analysis ${ }^{23}$ as displayed in table 1. Each investigator read and re-read the transcripts and manually identified the key themes from the data in addition to some example quotes to illustrate the main points. One of the authors (FCG) was masked to the emphasis on VF testing at this initial point of analysis, although became aware following a subsequent discussion about the key categories that had emerged during that first stage. The qualitative software package NVIVO V.10.2 (QSR International, Cambridge, Massachusetts, USA) was used to organise the thematic framework by refining and condensing the categories that had been manually identified and to identify additional themes for exploration. Any differences of opinion regarding the meaning of sentences or the importance of themes were discussed until a consensus was reached.

\section{FINDINGS}

Data were initially indexed according to themes central to the main research questions, such as opinions of the $\mathrm{VF}$ test, current experience regarding the frequency of VF testing and opinions about more frequent VF testing. Throughout the analysis, a number of additional themes emerged, often with their own subthemes; these generally related to specific areas perceived to affect the follow-up experience, and included points relating to clinical constraints (waiting times, booking appointments), travel to the clinic, the testing environment and aspects of patient-clinician communication. The themes and subthemes are summarised in figure 1 .

Table 1 Framework technique used for data analysis

\section{Framework technique}

1. Familiarisation Reading and re-reading the transcriptions

2. Identifying a Condense data into categories thematic framework

3. Indexing

4. Charting Codes systematically applied to the data

Re-arranging the data according to the thematic content in a way which allows for a cross case and within case analysis

5. Mapping and Interpretations and interpretation recommendations 


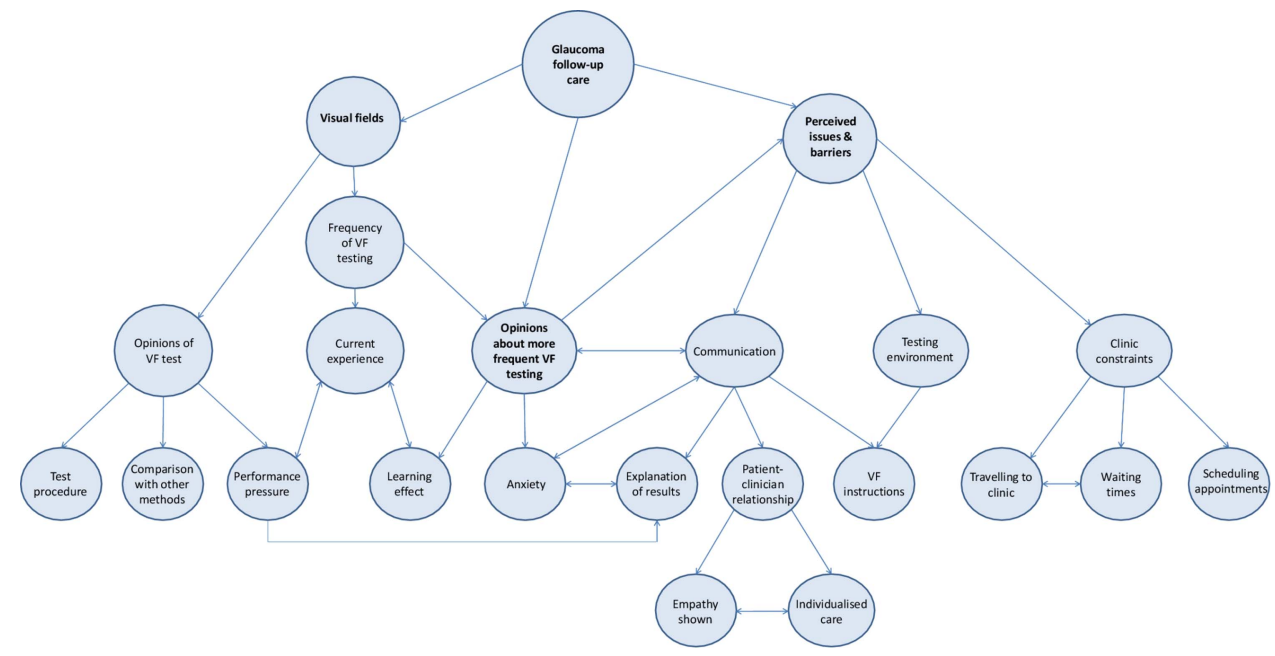

Figure 1 Coding tree showing main themes and subthemes that emerged from the analysis, and how the categories relate to each other.

Direct quotes taken from the transcripts are italicised. These quotes were examples chosen to illustrate the key themes that emerged from the focus groups. Excerpts are annotated with a pseudonym for the corresponding participant based on their gender (' $\mathrm{M}$ ' or ' $\mathrm{F}$ ') and the order in which they spoke in the interview. The location of the focus group and the session number ( 1 or 2 ) are also shown for each quote.

\section{VFs: opinions about testing}

\section{Test procedure}

Patients expressed a dislike for the VF test. They found the test time-consuming, old-fashioned and tiring.

Well the reason why I don't like them: I don't like the dark, I don't like confined spaces and I don't like having one eye closed and having to concentrate, even if it's for just a couple of minutes, because then my mind wanders... F1, Portsmouth 1

It seems a bit antiquated, pressing the buttons... it doesn't seem positive enough to me. F3 Norwich 2

I appreciate the need for it...but it's so time consuming. M2 London 1

\section{Performance pressure}

Many put pressure on themselves to perform the test well, as they felt there could be a lot riding on their performance.

There is pressure: I think it is because your eyes are so important for everyday living, that, you know, you're frightened to [not do well]. F2 Portsmouth 1

There was a general appreciation that such testing was vital to preserve their vision.

Well... obviously I'm very grateful that I'm being monitored all... F4 London 1 mine has been 10 years and you think, well how long will I have my sight? ... My mum had lost her sight by then, you know... F3 Norwich 2

\section{Comparison with other tests}

Patients found other tests used in their clinical monitoring, such as visual acuity, IOP measurement and imaging tests, less tiring and laborious. At the same time, some patients felt the VF test was more 'valuable', providing more reassurance that their condition was being investigated.

[with] the [imaging] there's just one person, one machine and you, and it's done and that's it, it's over... within minutes. F3 Norwich 2

they look in your eyes to measure your pressure but when you do that field test, they see more.... F1 London 2

\section{Frequency of VF testing \\ Current experience}

The VF tests were usually performed once or twice a year, either during or closely prior to the patient's general clinical appointment. Patients who visited the clinic more frequently would have a VF test at only some of their appointments. Some patients were often unaware as to whether they would have a VF test during their visit.

I mean they just say you're going to come for your next appointment in whatsoever, whatever time, but they don't say, ' $\mathrm{Oh}$, in that time you will be having a visual field check', so that you know that you are going to have to be that little bit longer... F2 Portsmouth 1

\section{Opinions about more testing}

When patients were asked whether they would be willing to visit the clinic for VF testing more frequently, there was a reluctant agreement. The test was viewed as a 
'necessary evil' and most were open to more frequent testing if the clinician felt it would enhance their prognosis, although there was scepticism as to how useful the test actually was.

If it was necessary. F2 Portsmouth 2

You'd get on with it. M1 Portsmouth 2

If it helps the cause so be it. M2 Portsmouth 2

I don't want to lose my sight, I'd come in whenever. F2 Portsmouth 2

If it holds it back for 10 years... I'm happy with another 10 years! M1 Norwich 2

That's a problem with glaucoma, you can't leave it for too long. M2 London 2

I suppose I'd accept it because I would hope that the reason for asking me was that they will get more information from that, which obviously deals with the whole problem but...I'm not really sure at all about how useful they are. I mean is it just statistics or whatever? ...I'm sure they're useful but I wonder in what proportion of use they are compared to, you know, looking in the eye and pressures and things.... F3 Norwich 2

Some patients associated more frequent testing with worsening vision; therefore, being asked to attend for more testing could lead to increased anxiety.

you'd think they've called me back 'cause it's going, deteriorating. But I mean if they said to do it, I've always done ... because they're doing the best for me... F3 Norwich 2

\section{Learning effect}

One recurrent topic regarding VF testing was issues relating to the learning effect, whereby performance improves with increased testing. Some suggested that more repeat testing would be helpful. However, the repeated tests may only be worthwhile if they took place at the beginning of their follow-up care.

interestingly I went and did one once and they said to me, "this has improved from the last time" and I said "well I think I'm just getting better at computer games" ... I think you do know what's coming and you can improve and I just feel more comfortable with doing it. F1 Norwich 1

I think to do a field test right at the beginning, and to take that as being the definitive field test is wrong... because I think you need to do a test and think, and revise it in your mind what you've done and then do it again. M1 Portsmouth 2

There was some debate about the period of time between VF tests.
I think you need to do a field test and then perhaps a month later do the second one. M1 Portsmouth 2

Well not if you have a long gap between them. F1 Norwich 2

I've got used to it now. F2 Norwich 2

I don't think it's any different really. F3 Norwich 2

\section{Perceived issues and barriers for successful follow-up care}

Some additional themes emerged during the analysis, highlighting a number of areas perceived to be important and potentially representing barriers to successful follow-up.

\section{Communication \\ VF instructions}

Regardless of how long they had been attending the glaucoma clinic, patients appreciated having the VF test procedure fully explained to them. It was rare for a staff member to stay with the patient throughout the test, but on the occasions it did happen, patients found the experience reassuring and felt the encouragement helped their performance.

They say, "Have you done this before?" You say "Yes". And that's it, you're left there and eventually they say, "Have you finished? M1 Portsmouth 2

I had one about three weeks ago and it was a young nurse and it was a completely different experience. She was professional, polite, kind; she told me exactly what they were doing... it was almost a pleasant experience. F1 Portsmouth 1

There was discussion about understanding the aspects of the testing procedure and how the procedure was explained. For example, some patients expressed uncertainty and felt test pressure would influence their results. Again, explanation and reassurance before and after the test helped.

The staff told me: "don't worry about missing [a light] because it'll come later", so you know you get a second chance. F1 Norwich 1

if in doubt press the button, don't you? F1 Portsmouth 2

\section{Explanation of results}

Most patients said they had to specifically enquire about their results to find out information about their vision and whether their condition had progressed since the last appointment. Some patients felt intimidated to ask the clinician for feedback as to how they had performed, feeling they were being a nuisance or wasting the clinician's time. 
They never discuss the result of the field test unless I ask... M2 London 2

My wife always says "how did you get on?" and I say "I don't know", and that's one of the problems. M2 Portsmouth 2

I don't think they've got time to listen to you, or they don't appear to, and I don't know whether they would listen.... You feel pathetic asking these questions. F3 Portsmouth 1

Patients may be more inclined to have the VF tests more frequently should they be informed clearly about what the results indicate about their prognosis.

I don't mind how many times I do it providing I get a result of the test at that time compared to what the previous one was. Is there any improvement? Is there any downgrade? M1 Portsmouth 1

\section{The patient-clinician relationship}

The quality of relationship with the clinical staff and aspects of patient-clinician communication also emerged as key factors influencing perceptions of the follow-up process.

An apparent lack of personalised care caused unease; there was a sentiment that sometimes the clinician simply looked at the eyes and failed to consider the person's individual needs.

You're not a person, you know, you've just got eyes, they're just going to deal with that and that's it. F3 Portsmouth 1

The experience was seen to be much more bearable if they felt the staff member dealing with them was empathic.

Even buying a chop, you know: if the butcher's interested, it helps doesn't it? M3 Norwich 1

The opportunity to spend more time with their consultant ophthalmologist was a key factor that influenced whether or not patients were open to visiting the clinic more frequently.

Not [just] for the field test... But I wouldn't mind coming in more to see the doctor. M2 London 2

\section{Testing environment}

The testing environment was another important theme. The dark room, especially if it was warm, made focusing on the tests difficult. Patients felt they performed better in the morning when they were more alert. Ambient noise in the room made it difficult to concentrate; staff members talking and performing the test at the same time as several other patients all had deleterious effects.
I will also say that the staff chatter a lot, which is difficult for concentration; the doors open and close, there's a lot of noise. F1 Norwich 1

I find it difficult sometimes when people [move] about behind you... M1 London 1

The times that I've had the visual field test done in a room where there's just one [machine], I felt more confident to do it; it was much quieter and more relaxed and it seemed to be a lot quicker too. F3 Norwich 2

I think having the quieter atmosphere would generally help I'm sure....just that feeling of slight calm, you can relax more and then it probably would be a lot quicker because maybe you're not going to miss as many [lights] as you haven't got other distractions. F3 Norwich 2.

The idea was raised that routine VF testing could be carried out in a more convenient location. Some patients had previously visited a local optometrist to carry out a VF test for the purpose of assessing their legal fitness to drive. On the positive side, patients liked the convenience of doing so and described a better testing environment. Conversely, they questioned the competency of the staff, the quality of the equipment and the information trail back to the hospital.

The principle of having routine tests done locally is acceptable providing they are trained. M1 London 1

That way you would be there, dealt with by people you know probably more intimately...you're in a more relaxed environment... M1 Norwich 1

I would be concerned about how often the machine was calibrated to get an accurate reading. M2 London 1

Is the information going back to where it matters in my notes? Things do get lost, and will someone actually look at the test? M1 London 2

Some felt that they had built up a level of trust with the hospital eye service and would therefore prefer to have VFs conducted in this environment.

I've been here for quite a while now and I like coming to them: I don't want to go anywhere else. F1 London 2

I would feel the same because it's a matter of trust. M2 London 2

\section{Clinic constraints}

\section{Waiting times}

Waiting times were a major concern at all locations. The standard time taken per visit was estimated to be $2 \mathrm{~h}$, although the wait was often unpredictable. Established patients were used to wait and tried not to let it affect them but they still found the system frustrating. Patients were scared of missing their slots and, therefore, would not leave their seat in the waiting area. 
No way I'm going to nip off ... especially as now I'm on my own, no way.... just even nipping off to the [bathroom] because you think, 'He's bound to call me. I can sit here for an hour and he'll call me the minute I go to the [bathroom]. F2 Portsmouth 1

Although it was repeatedly acknowledged that the clinics were very busy, which had the knock-on effect of increased waiting times, patients felt that they were getting adequate treatment overall. It was suggested that there was a trade-off between longer waiting times and higher quality treatment:

I think that's a very fair price to pay for the fact that you're being dealt with in a UK centre of excellence. There's a trade-off in that you're getting state of the art treatment but the price is you've got to sit around for it. M1 London 1

\section{Travelling to the clinic}

Several subthemes emerged including issues with long distances to travel, avoiding rush hours, travel costs and travelling alone.

I think the problem is because I live nearly an hour away, for me the nearest hospital is an hour away... F2 Norwich 2

Taxi is the only way I can do it now. You know, I can get to the station by bus and possibly with help to get on the train but it's not easy.... It's horrific, frightening. M2 London 1

Tiring journeys to the clinic and late clinic appointments were also sometimes perceived to have a negative effect on VF test performance.

I think if you did the eye check later in the day, you know, if your eyes were tired, it might make you feel [that you] wouldn't see so well...F2 Portsmouth 1

\section{Scheduling appointments}

The scheduling of appointments was a major concern: often the systems were so overbooked that patients were unable to make their next appointment at their clinic visit.

You can only make an appointment six weeks in advance. You used to get a twelve month appointment letter just after you had been for an appointment; now its six weeks before you are due. M2 Norwich 1

Often patients would receive an appointment only to have it cancelled just before the clinic was due to take place. This was not only frustrating to people who had made arrangements for their appointment, such as asking a friend to accompany them or arranging cover for sick spouses, but also it caused concern that their appointment was to be at a much later date than the clinician had originally requested.
So if you've been given a six month appointment and it's cancelled, and you're not given another one, you ring up and then they say "oh we can't give you an appointment now until October". That was 10 months. Now if your consultant says 6 [months] and it's 10 and something's gone wrong with your vision in between, you have no way of telling. F2 Portsmouth 2

\section{PATIENT RECOMMENDATIONS}

At the end of the focus groups, patients were asked to recommend changes to improve their follow-up care. The recommendations were similar across all locations and the most popular suggestions are displayed in table 2.

\section{DISCUSSION}

Data from this study support evidence from elsewhere that patients find VF testing more laborious and demanding than other vision tests. ${ }^{20}$ Nevertheless, patients were willing to complete more VF tests on the guidance of their clinician, as ultimately they were prepared to do whatever it took to preserve their vision. Thus, patients may tolerate more frequent VF testing during the first 2 years of their follow-up care as recommended by the research literature ${ }^{45}$ and some clinical guidelines. $^{24}$ Patients commented that it took time to feel comfortable with the test procedure, and that multiple attempts were needed to gain an accurate representation of their vision. These viewpoints complement the existing evidence showing that performance can improve considerably during follow-up due to gaining experience with the testing process. ${ }^{25}$

There were, however, a number of additional themes that emerged from the data which identified areas that could represent potential barriers to successful glaucoma monitoring. Patients felt that the environment in which they completed the VF test was linked to how well they were able to perform the task, with staff members talking loudly, the number of people in the room and the time of day all listed as important interfering factors. These views coincide with other evidence showing that the environment, the technician and the time of day do

Table 2 Patient recommendations for improving follow-up care

\section{Patient recommendations}

1. Less waiting and clinics running to time

2. Flexible booking and changing of appointments

3. To have a calmer, quieter environment in the visual field room with fewer people doing the test at the same time

4. To modernise the visual field test

5. To have more continuity of care by seeing the same clinician at each visit

6. To receive better communication from the clinician 
have a significant influence on measurement variability from VF tests. $^{26}$ Fatigue, a topic mentioned frequently throughout the discussions, has also been shown to affect performance as test duration increases. ${ }^{27}$

Patients highlighted the importance of effective task communication for influencing their VF test performance. Prior evidence has shown that the quality of instruction given before the VF test can significantly affect subsequent estimations of VF defect severity. ${ }^{28} 29$ Patients also felt that it was essential to have the task explained to them properly, even if they had been attending clinics for some time. Having a staff member in the room while they carried out the test was found to be reassuring. These findings reiterate the idea that ensuring the task demands are communicated clearly and effectively before every VF test, and being on hand to alleviate any concerns or questions that the patient may have, may help maximise the quality of the data gained from the assessment. ${ }^{29-31}$

Other discussion points relating to communication were also raised repeatedly throughout the focus groups. Patients felt that many clinicians treated them as an 'eye' rather than a person, with those staff members who took a more individualistic and empathic approach viewed favourably. Notably, patients felt that they had to ask explicitly about their results in order to learn details about their own condition. Evidence has shown that that the patient's and clinician's views of their condition are not always aligned, which may be due to miscommunication or misinterpretation of key information on both parts. $^{32}$ By explaining the results in a clear, simple and concise manner, the patient will inevitably improve their understanding of their condition, which in turn could influence how well they respond to important aspects of their follow-up care. For instance, it has been shown that the way in which clinicians communicate with the patient can influence future adherence to medication. ${ }^{32}$ It has been suggested that clinicians underestimate the importance of effective communication to the patient, ${ }^{11}$ and in one study, examining the patient's expectations for eye care, the emphasised areas were all related to communication and interpersonal manner. ${ }^{33}$ Providing better information about the purpose of VF testing, what is required of the patient, and its results and general prognosis could be vital for improving attendance for VF tests or for the subsequent quality of data obtained. Perhaps, developers of SAP ought to think about ways in which the complex measurement of the $\mathrm{VF}$ could be easily presented and communicated to patients. It is important to note that some patients associated more frequent testing with worsening vision, which caused some distress. Thus, should patients require more frequent tests at some point in their care, it is also vital to involve the patient and explain reasons for the decision.

Excessive waiting times and difficultly booking appointments were also major concerns. In particular, patients worried that appointment cancellations could extend the interval between tests beyond what was recommended by the clinician, therefore leaving them exposed to undetected disease progression. It is known that while clinicians select appropriate monitoring intervals, hospital-initiated rescheduling is a major challenge to appropriate follow-up. ${ }^{74} 35$ Moreover, it was typical for patients to wait at the clinic for hours in order to complete multiple vision tests, causing frustration and tiredness which some perceived to influence their subsequent performance. Potential solutions could involve conducting only the VF test during short independent appointment slots, or carrying out tests at a more convenient location. However, such strategies would involve further investigation as to their overall cost-effectiveness and should address other associated practicalities such as travel (a significant contributor to total patient $\operatorname{costs}^{36}$ ) and the information trail back to the hospital.

Previous research has relied on statistical analysis or computer simulations to help determine the most effective VF monitoring strategies for patients with glaucoma. This is the first study to use qualitative methods to investigate the patient's own perspective on their follow-up. Studies focusing on the patient's perspective in glaucoma, particularly with regard to the perceived effects of the disease on their day-to-day activities, have typically relied on questionnaires. ${ }^{13}$ However, questionnaire responses can be restricted by the wording of the items and provide little opportunity for clarification or elaboration. This study allowed individuals to contextualise their experiences and expand on particular points and themes, encouraging discussion about topics certain patient may not have otherwise introduced or attributed to glaucoma without the encouragement of another. ${ }^{18}$ The notion of the 'expert patient' is beginning to be endorsed with regard to other chronic conditions, with focus groups demonstrating potential as a forum for the development of more effective management strategies. ${ }^{37-39}$ Furthermore, patient groups have aided the development of health education programmes for age-related macular degeneration. ${ }^{40} \mathrm{~A}$ systematic review of patient-centred randomised controlled trials suggests there may be some benefits associated with involving patients with chronic disease in programmes geared towards better educating service users and devising general training for health professionals. ${ }^{41}$ Future work that encourages more patient's involvement may therefore help devise the optimal strategies for glaucoma follow-up and also help better inform patients and health professionals about the condition.

This study has its limitations with findings attached to the viewpoints of the groups who took part. Efforts were taken to reduce bias by involving multiple research sites; however, these were all geographically limited to the South of England and (excluding the London groups) involved patients of Caucasian ethnicity. Therefore, the findings may not necessarily translate to a wider population. Moreover, initial patient selection was made on recommendation of consultants at the clinics and our 
selection process did not monitor reasons for nonparticipation. People who choose to volunteer for focus groups are likely to be articulate and confident; they may also be more motivated to take part due to having more severe disease or holding strong opinions about a certain area of their care. Furthermore, participants were aged 60 years and older-younger service users may have differing views and experiences that also warrant investigation. The study was initially designed to involve six focus groups across three locations and so no direct decision was taken to cease data collection; however, similar themes and subthemes continued to emerge in the latter focus groups and so it is likely that 'data saturation' was achieved. Furthermore, some of the focus groups were small (one consisting of only 3 participants) due to late cancellations but this is not a major limitation due to the number of focus groups that took place. ${ }^{42}$ Also, some biases could have been introduced during interview and analysis due to the preconceived ideas held by the experimenters about the areas of importance, although care was taken to adhere to expected practice by following the COREQ checklist for focus group research. ${ }^{22}$

A number of important themes did emerge that give an insight into clinic visits and VFs from the patient's perspective, and could help inform patient-centred care in glaucoma. Although patients appeared frustrated by a number of aspects of their follow-up, they ultimately accepted that some compromises had to be made in order to save their eyesight. Some of the viewpoints illustrated in the focus group discussions may in part explain why research-supported guidelines about more frequent VF testing are not being implemented effectively in clinical practice. A holistic approach that embraces patients' opinions may therefore be vital to help devise the most effective strategies for follow-up care in this chronic disease.

\section{Conclusion}

This is the first study to use qualitative methods to examine patient's opinion about the glaucoma clinic experience and VF tests. Although patients found the VF test onerous, they accepted it was important to their overall vision assessment. However, a number of actionable points were raised which were perceived to impact the effectiveness of follow-up care, including distracting testing environments, and hospital constraints relating to excessive waiting times and appointment booking. Some patients also expressed particular concerns about the VF technology used and the quality of test instructions. Anxiety associated with increased testing in the absence of clinical explanation was another theme. Ensuring that glaucoma monitoring is carried out as clinically and cost-effectively as possible will inevitably require the confidence and cooperation of the patient. Addressing some or all of the perceived barriers highlighted in this study should help deliver more efficient strategies for VF monitoring in glaucoma.

Acknowledgements The authors would like to thank The Queen Alexandra Hospital NHS Trust in Portsmouth; Norfolk and Norwich University Hospital NHS Foundation Trust in Norwich and Moorfields Eye Hospital NHS Foundation Trust in London for accommodating the focus groups. They also thank Consultant Ophthalmologists Mr James Kirwan, Mr David Broadway and Professor David Garway-Heath for their advice and assistance with participant recruitment. They are also grateful to all patients who participated in the focus groups, including Carol Bronze and Julia Brazear for their help with the pilot stages of the project.

Contributors FCG conducted data analysis, and wrote and edited the manuscript. HB conducted focus groups and data analysis, and wrote the manuscript. DPC conceived and designed study, and reviewed and edited the manuscript.

Funding This work was funded by the National Institute of Health Research (NIHR), Health Services and Delivery Research programme (project number 10/2000/68). FCG's research position is also partially funded by grants from the International Glaucoma Association and Fight For Sight. The views expressed are those of the authors or interviewees and not necessarily those of the NHS, the NIHR or the Department of Health.

Competing interests None.

Patient consent Obtained.

Ethics approval The study received approval from a National Health Service (NHS) National Research Ethics Service (NRES) committee (North West REC Centre), and R\&E approval at participating institutions (Moorfields Eye Hospital NHS Foundation Trust, Queen Alexandra Hospital NHS Trust; Norfolk and Norwich University Hospital NHS Foundation Trust).

Provenance and peer review Not commissioned; externally peer reviewed.

Data sharing statement Copies of the topic guide and participant information sheet can be obtained by emailing the corresponding author.

Open Access This is an Open Access article distributed in accordance with the Creative Commons Attribution Non Commercial (CC BY-NC 3.0) license, which permits others to distribute, remix, adapt, build upon this work noncommercially, and license their derivative works on different terms, provided the original work is properly cited and the use is non-commercial. See: http:// creativecommons.org/licenses/by-nc/3.0/

\section{REFERENCES}

1. National Institute for Health and Care Excellence. Glaucoma: diagnosis and management of chronic open angle glaucoma and ocular hypertension. April 2009. http://www.nice.org.uk/nicemedia/ live/12145/43887/43887.pdf (accessed 15 Jul 2013).

2. Burr JM, Mowatt G, Hernández RA, et al. The clinical effectiveness and cost-effectiveness of screening for open angle glaucoma: a systematic review and economic evaluation. Health Technol Assess 2007;11:1-190.

3. Heijl A, Bengtsson B, Hyman L, et al. Natural history of open-angle glaucoma. Ophthalmology 2009;116:2271-6. http://www.ncbi.nlm. nih.gov/pubmed/19854514(12):2271-6

4. Chauhan BC, Garway-Heath DF, Goni FJ, et al. Practical recommendations for measuring rates of visual field change in glaucoma. Br J Ophthalmol 2008;92:569-73.

5. Gardiner SK, Crabb DP. Frequency of testing for detecting visual field progression. Br J Ophthalmol 2002;86:560-4.

6. Crabb DP, Garway-Heath DF. Intervals between visual field tests when monitoring the glaucomatous patient: wait-and-see approach. Invest Ophthalmol Vis Sci 2012;53:2770-6.

7. Fung SS, Lemer C, Russell RA, et al. Are practical recommendations practiced? A national multi-centre cross-sectional study on frequency of visual field testing in glaucoma. Br J Ophthalmol 2013;97:843-7.

8. Malik R, Baker H, Russell RA, et al. A survey of attitudes of glaucoma subspecialists in England and Wales to visual field test intervals in relation to NICE guidelines. BMJ Open 2013;3:e002067.

9. Lacy NL, Paulman A, Reuter MD, et al. Why we don't come: patient perceptions on no-shows. Ann Fam Med 2004;2:541-5. 
10. Owsley C, McGwin G, Scilley K, et al. Perceived barriers to care and attitudes about vision and eye care: focus groups with older African Americans and eye care providers. Invest Ophthalmol Vis Sci 2006; 47:2797-802.

11. Laine C, Davidoff F, Lewis CE, et al. Important elements of outpatient care: a comparison of patients' and physicians' opinions. Ann Intern Med 1996;125:640-5.

12. Brown GC, Brown MM, Sharma S. Difference between ophthalmologists' and patients' perceptions of quality of life associated with age-related macular degeneration. Can $J$ Ophthalmol 2000;35:127-33.

13. Glen FC, Crabb DP, Garway-Heath DF. The direction of research into visual disability and quality of life in glaucoma. BMC Ophthalmol 2011;11:19.

14. Ormel J, Kempen GI, Penninx BW, et al. Chronic medical conditions and mental health in older people: disability and psychosocial resources mediate specific mental health effects. Psychol Med 1997;27:1065-77.

15. Friedman SM, Munoz B, Rubin GS, et al. Characteristics of discrepancies between self-reported visual function and measured reading speed. Salisbury Eye Evaluation Project Team. Invest Ophthalmol Vis Sci 1999;40:858-64.

16. Kitzinger J. Qualitative research: introducing focus groups. BMJ 1995;311:299-302.

17. Lacey J, Cate H, Broadway D. Barriers to adherence with glaucoma medications: a qualitative research study. Eye 2008;23:924-32.

18. Green J, Siddall H, Murdoch I. Learning to live with glaucoma: a qualitative study of diagnosis and the impact of sight loss. Soc Sci Med 2002;55:257-67.

19. Prior M, Francis JJ, Azuara-Blanco A, et al. Why do people present late with advanced glaucoma? A qualitative interview study. $\mathrm{Br} \mathrm{J}$ Ophthalmol 2013;97:1574-8.

20. Gardiner SK, Demirel S. Assessment of patient opinions of different clinical tests used in the management of glaucoma. Ophthalmology 2008;115:2127-31.

21. Patel D, Baker H, Murdoch I. Barriers to uptake of eye care services by the Indian population living in Ealing, west London. Health Educ $J$ 2006;65:267-76.

22. Tong A, Sainsbury P, Craig J. Consolidated criteria for reporting qualitative research (COREQ): a 32-item checklist for interviews and focus groups. Int J Qual Health Care 2007;19:349-57.

23. Pope C, Ziebland S, Mays N. Qualitative research in health care: analysing qualitative data. BMJ 2000;320:114.

24. European Glaucoma Society. Terminology and guidelines for glaucoma. 3rd edn. Savona, Italy: Editrice Dogma 2008, 2008.

25. Wild JM, Searle AET, Dengler-Harles M, et al. Long-term follow-up of baseline learning and fatigue effects in the automated perimetry of glaucoma and ocular hypertensive patients. Acta Ophthalmol $1991 ; 69: 210-16$
26. Junoy Montolio FG, Wesselink C, Gordijn M, et al. Factors that influence standard automated perimetry test results in glaucoma: test reliability, technician experience, time of day, and season. Invest Ophthalmol Vis Sci 2012;53:7010-17.

27. Henson DB, Emuh T. Monitoring vigilance during perimetry by using pupillography. Invest Ophthalmol Vis Sci 2010;51:3540-3.

28. Kutzko KE, Brito CF, Wall M. Effect of instructions on conventional automated perimetry. Invest Ophthalmol Vis Sci 2000;41:2006-13.

29. Sherafat $H$, Spry PGD, Waldock $A$, et al. Effect of a patient training video on visual field test reliability. Br J Ophthalmol 2003;87:153-6.

30. Heijl A, Asman P. Pitfalls of automated perimetry in glaucoma diagnosis. Curr Opin Ophthalmol 1995;6:46-51.

31. Van Coevorden RE, Mills RP, Chen YY, et al. Continuous visual field test supervision may not always be necessary. Ophthalmology 1999;106:178-81

32. Friedman DS, Hahn SR, Quigley HA, et al. Doctor-patient communication in glaucoma care: analysis of videotaped encounters in community-based office practice. Ophthalmology 2009;116:2277-85 e1-3.

33. Dawn AG, Santiago-Turla C, Lee PP. Patient expectations regarding eye care: focus group results. Arch Ophthalmol 2003:121:762-8.

34. Tatham A, Murdoch I. The effect of appointment rescheduling on monitoring interval and patient attendance in the glaucoma outpatient clinic. Eye (Lond) 2012;26:729-33.

35. Agency NPS. Preventing delay to follow-up for patients with glaucoma. Rapid Response Report, 2009.

36. Sharma A, Jofre-Bonet M, Panca M, et al. Hospital-based glaucoma clinics: what are the costs to patients? Eye 2009;24:999-1005.

37. Wilson PM, Kendall S, Brooks F. The Expert Patients Programme: a paradox of patient empowerment and medical dominance. Health Soc Care Community 2007;15:426-38.

38. Holman $\mathrm{H}$, Lorig K. Patients as partners in managing chronic disease: partnership is a prerequisite for effective and efficient health care. BMJ 2000;320:526.

39. Holman H, Lorig K. Patient self-management: a key to effectiveness and efficiency in care of chronic disease. Public Health Rep 2004:119:239.

40. Dahlin-Ivanoff S, Klepp K, Sjöstrand J. Development of a health education programme for elderly with age-related macular degeneration: a focus group study. Patient Educ Couns 1998;34:63-73.

41. McMillan SS, Kendall E, Sav A, et al. Patient-centered approaches to health care: a systematic review of randomized controlled trials. Med Care Res Rev 2013;70:567-96.

42. Carlsen B, Glenton C. What about N? A methodological study of sample-size reporting in focus group studies. BMC Med Res Methodol 2011:11:26. 